The spectrum is more or less a camouflage and is not necessarily a secure indicator of $T_{\text {eff }}$ and thus of the bolometric correction.

Petrie: The spectral types are estimated, but there are $B-V$ data and they are plotted on the HR diagram in $B-V$.

Underhill: The colour $(B-V)$ is not a more reliable indicator of $T_{\text {eff }}$ than the spectrum is. The difficulty is to relate the strength of the strong lines in a unique manner to $T_{\text {eff. }}$ My suspicions are that the relation is different in the case of luminous stars from the relation valid for main-sequence stars, and that for given relative line strengths, $T_{\text {eff }}$ is smaller in the luminous stars.

Popper: $\mathrm{H}_{\gamma}$ or $\mathrm{H}_{\beta}$ equivalent widths correlate well with luminosity with small scatter. $B-V$ correlates well with $M_{\mathrm{v}}$ in cluster $C-M$ diagrams. So perhaps these quantities depend on fundamental properties of the star, rather than on superficial effects as suggested by Miss Underhill.

Kraft: Regarding binaries with one member 6 magnitudes brighter than the companion and both near the main sequence, cannot the nuclear time-scale for the primary, and contraction time-scale for the secondary be made compatible by using the Hayashi tracks for the secondary?

Batten: The diagram is simply a plot of the observed positions of the stars in the HR diagram. Our idea was that the expected difference of evolutionary stages between the two components of such systems is very large on any reasonable time-scale, but this does not seem to be the case.

Schwarzschild: What worries me most in Dr Petrie's diagram are the two binaries with the primaries on the main sequence but the fainter components above the main sequence by about I magnitude, at absolute magnitude around +3 or +4 . Are these observations so sure that the fainter components cannot be pushed onto the main sequence?

Batten: One of these stars is one for which the evidence of physical connection is still weak. There is some evidence, however, and these systems remind one strongly of the much closer semi-detached systems with subgiant secondaries.

Schwarzschild: Are they wide pairs?

Batten: Yes, indeed.

\title{
3. PHYSICAL DATA FROM NORMAL BINARIES
}

\section{M. Popper}

\section{INTRODUCTION}

Starting with his pioneering studies of $U$ Cephei and $\beta$ Lyrae, Prof. Struve and a number of able collaborators, several of whom are here today, demonstrated that superficial effects are present in many close binaries that influence the spectrographic observations in such a way that the true orbital velocity variations, in particular, may not always be readily obtainable. Struve's principal interest was in studying these effects in order to understand their physical nature, their causes, and their possible influence on the evolutionary history of the system. Later speakers will emphasize these aspects of the topic. 
The title of this talk refers to 'normal' binaries. There are not, of course, two classes of close binaries, normal and peculiar. Despite whatever disturbing effects may be present in its spectrum or light curve, every star has a mass, a luminosity, and a size. It is true that for many close binaries the superficial effects may prevent us from obtaining reliable physical data, and for this reason they are not likely to be included in my discussion. But so long as a star is free to adjust its luminosity and its size in accordance with equilibrium considerations of stellar structure and is not dynamically trapped within the critical zero-velocity surface of the system, it will not be excluded as a matter of principle. From another standpoint, no close binary is normal in that each of them must have had an early evolutionary history quite different from that of a single star or member of a wide binary.

In the time at my disposal I will treat principally problems of obtaining fundamental data from eclipsing binaries, with problems of visual binaries referred to in passing. Eclipsing binaries relate more closely to the subject of this Joint Discussion, and I am more familiar with them.

\section{SELECTION OF SYSTEMS}

Eclipsing binary analysis yields mass and radius directly. Visual binary analysis yields mass and absolute magnitude. Of these fundamental properties, mass, being proportional to the third power of the linear dimensions of the system, is the most difficult to obtain with precision. Moreover, in comparing data from different systems ( $m-L$ relation) or in comparing observations with theory (stellar structure and evolution), the mass is the parameter upon which the other properties of a star depend most sensitively (e.g. $L \propto m^{4}$ ). From these considerations it follows that to determine whether a star of known luminosity falls on a given mass-luminosity relation within 0.5 magnitude, for example, the amplitudes of the radial-velocity variation, if the star is an eclipsing binary, must be known to about 4 per cent. As another example, to determine whether an eclipsing binary falls closer to the 'Sun-Sirius' or Hyades mass-luminosity relation as delineated by Eggen (I), the radial-velocity amplitudes need to be known to about 5 per cent. Thus in selecting systems to be included in a critical list of masses, one requires that both accidental and systematic errors in the velocities be known and be small. There are relatively few systems for which the accuracy indicated above is met. For certain purposes, of course, one can tolerate larger uncertainties. In the earlier B types and for stars well above the main sequence, the demands at the present time are less rigid.

Thus far the requirements on the velocity variations of eclipsing binaries have been stressed. The requirements for mass and radius determination upon the orbital inclinations and fractional radii obtained from light curves are much less stringent. The light variations between minima must not be too large relative to the depths of minima, and the minima must be such that the fractional radii are determined to an accuracy of 20 per cent or better for most problems. Uncertainties in the temperature scale, used in computing the luminosities of eclipsing binaries, have been discussed elsewhere (2). Thus an eclipsing binary may be considered a promising candidate if, first, the light curve is of such a character as to yield a determinate solution for the inclination and fractional radii, and second, the lines of both components are visible clearly and unblended in the spectrum. Fulfilment of the latter requirement is, of course, dependent upon the dispersion and resolution of the spectrograms employed and may depend also on the region of the spectrum studied. It is my opinion that, except for stars of mass larger than about 4 solar masses, lower dispersions than correspond to $20 \AA / \mathrm{mm}$ are inadequate, and $10 \AA / \mathrm{mm}$ or higher is to be preferred.

To say just a word about criteria of selection of visual binaries: The corresponding problems in mass determination concern the precision of parallaxes and accidental and systematic errors in the scales of visual orbits. Eggen (I) has stressed the importance of this last item. 
The best eclipsing systems for the purposes under discussion are those with nearly equal components, with eclipses 0.5 magnitude or more deep, and with relatively little tidal distortion. Such systems later than spectral type B8 include RX Herculis*, AR Aurigae*, V45I Ophiuchi, V805 Aquilae, WW Aurigae*, TX Herculis, ZZ Bootis, EI Cephei, CD Tauri, VZ Hydrae, WZ Ophiuchi*, UV Leonis*, and YY Geminorum*, listed in approximate order of decreasing temperature. An asterisk(*) indicates a system for which both spectrographic and photometric analyses are probably adequate. For the rest, one or the other is lacking, often a definitive light curve in a standardized color system. There may be additional suitable systems as yet undiscovered, particularly in the southern sky. Somewhat less satisfactory systems are those with two main-sequence stars with quite different depths of minima. In these cases the lines of the second component are difficult to measure. Among them may be included CM Lacertae, V477 Cygni, RR Lyncis, TV Ceti, and MY Cygni which are under investigation spectrographically, and some of which, particularly TV Ceti and MY Cygni, lack modern light curves.

\section{PROBLEMS IN ANALYSIS OF OBSERVATIONAL DATA}

Here emphasis is on problems associated with evaluating the radial-velocity amplitudes, $K_{1}$ and $K_{2}$ for eclipsing binaries. Table $\mathrm{r}$ shows for a number of systems the change in log $m$ (where $m$ is the stellar mass), relative to earlier evaluations, obtained from changes in the values of $K$ resulting from improved material and analysis. $\Delta \log m$ is the improved value minus the previously published value.

Table I

$\begin{array}{lcclcc}\text { Star } & \text { Spectrum } & \Delta \log m & \text { Star } & \text { Spectrum } & \Delta \log m \\ \text { RX Her } & \mathrm{A} & +0.1 \mathrm{I} & \text { WZ Oph } & \mathrm{F} & -0.14 \\ \text { RX Her } & \mathrm{A} & +\cdot 10 & \text { WZ Oph } & \mathrm{F} & -\cdot 10 \\ & \mathrm{~F} & -\cdot 10 & \text { UV Leo } & \mathrm{G} & -\cdot 12 \\ \text { Z Her } & \mathrm{K} & -.08 & \text { UV Leo } & \text { G } & -.12 \\ \text { Z Her } & \mathrm{F} & -.14 & \zeta \text { Aur } & \mathrm{B} & -.25 \\ & \mathrm{~K} & -.08 & \zeta \text { Aur } & \mathrm{K} & -.4\end{array}$

To put these values in perspective, 0.2 is the difference in $\log m$ between Hyades stars and the Sun, for equal luminosity, so that the corrections average more than half that amount. Fifteen years ago, a solar type star $\left(\mathrm{G}_{2}\right)$ of absolute magnitude +4.4 and mass $1 \cdot 3$ solar masses (UV Leo) would not have (and did not) attract particular attention. But our knowledge of stellar structure and evolution has advanced to the point where such a star would be considered either an object of hitherto unknown type or an example of unreliable data. The latter turns out to have been the case.

The causes of systematic error in the velocity variations have included blending of the wings of hydrogen lines; the presence of unresolved interstellar lines (on lower dispersion (3)); difficulty in finding lines of one component not blended with the oppositely displaced lines of the other component $(4,5)$; inadequate dispersion causing overestimates of $K_{1}+K_{2}$ even for apparently sharp, separated lines (6); distortion of the profile of a broad line by sharp lines of the other component (7). These effects are illustrated with examples, principally from published material. If such systematic effects are eliminated and the lines are of good quality, a relatively small number of spectrograms will, in favorable cases, suffice to determine the velocity amplitudes with the required precision. 
In view of such findings, the reliability of the masses and radii of eclipsing systems requires reassessment, i.e. for which systems are the published spectrographic results trustworthy, for which systems are additional observations required, and what new systems appear promising.

Requirements on the analysis of the light curves have been referred to briefly earlier. I should just like to add here that for evaluation of the position of a star in the HR diagram, the colors of the components need to be determined on a well-standardized system and corrected for interstellar reddening. The color is needed both for the abscissa in the HR diagram and for the temperature required in evaluating the luminosity from $L \propto R^{2} T_{\text {eff }}{ }^{4}$. Alternative methods of evaluating the luminosity will be referred to in the next section.

The $\mathrm{O}$ and $\mathrm{B}$ stars present special problems, among which are the following. To what extent has blending of the wings of the hydrogen lines affected the published values of $K_{1}+K_{2}$ ? Why do the values of $K_{1}+K_{2}$ for the favorable system U Oph published by Plaskett (8) and by Abrami (9) differ so significantly? What is the reason for the great differences in $K_{1}+K_{2}$ obtained from different lines by Ovenden (ro) in the spectrum of 57 Cygni? Until these questions are answered, the status of the masses and radii of $O$ and $B$ stars is open to some reservations. Additional problems for stars of these types are that the light curves of systems favorable from the spectroscopic standpoint tend to be unfavorable for analysis (tidal distortion, indeterminate solution); and the temperature scale, and hence the bolometric luminosities, await definitive observation of ultraviolet fluxes and their interpretation.

\section{ALTERNATIVE APPROACHES}

The 'traditional' methods of evaluating masses and luminosities, implied in the preceding discussion and upon which most existing compilations have been based, are as follows. For eclipsing binaries, masses are obtained from the period, $K_{1}, K_{2}$, and the orbital inclination (also the eccentricity if appreciably different from zero); luminosities are evaluated from absolute radii and effective temperatures, the latter having been obtained from accurate colors, preferably, and/or spectral types. For visual binaries, masses are obtained from orbital analyses, trigonometric parallaxes, and mass ratios; luminosities are evaluated from apparent magnitudes, trigonometric parallaxes, and bolometric corrections. One may add among the traditional methods the special cases of cluster parallaxes for well-established members of the Hyades cluster and systems ( $\alpha$ Aur) with both visual and spectroscopic orbits of precision. This morning Dr Petrie discussed further application of the latter method.

In the case of an eclipsing binary there appears to be no alternative approach for mass determination. There are, however, alternative methods of determining luminosities that have the advantage of not requiring evaluation of the radii and effective temperatures. These methods, which lead to $M_{n}$ rather than to $M_{\text {bol }}$, include first, evaluation of absolute magnitudes by spectrophotometry or interference-filter photometry, criteria being calibrated from stars of known luminosity. At Saltsjöbaden recently McNamara reported on results from H $\beta$ photometry of eclipsing binaries of earlier types. At the 1964 Flagstaff meeting of the American Astronomical Society, Koch, Olson, and Yoss reported on spectrophotometric measurements of a number of lines in early-type eclipsing systems, the intensities and line ratios having been calibrated from MK standards. With the recent improvement of the calibration of luminosities from $\mathrm{H}$ lines by Petrie and others, these methods have considerable promise. But the effects of blending of the lines and overlapping of the continuous spectra of the two components must be carefully allowed for and the measurements must be adequately calibrated in terms of luminosities to an accuracy of a few tenths of a magnitude-a demanding task.

Two additional methods of luminosity determination have been employed for a few eclipsing binaries by Eggen ( $\mathbf{x}$ ), namely 'photometric parallaxes' obtained from the color and magnitude of a visual companion assumed to lie on the main sequence, and membership in a 'moving 
group'. Eggen has discussed the conditions (brighter star near the main sequence, $B-V$ of the fainter companion between +0.6 and +0.8$)$ under which photometric parallaxes are useful. The moving group method in addition to the uncertainty as to actual membership of the star in the group, has the disadvantage of requiring that the group velocity vector and the proper motion and radial velocity of the star be known with higher precision than can, in general, be expected to yield absolute magnitudes to within \pm 0.5 magnitude. One may refer further to Eggen's inclusion of binaries for which the component of motion perpendicular to the galactic plane differs by as much as $20 \mathrm{~km} / \mathrm{sec}$ from that of the assumed group motion.

We turn next to alternative approaches for visual binaries. Here parallaxes are required for evaluation of both masses and luminosities, though the effect of uncertainties of the parallaxes on the masses is much the more important. Spectroscopic parallaxes are clearly inadequate for determining the linear dimensions of orbits to adequate precision. Methods alternative to trigonometric parallaxes proposed by Eggen have been referred to above, namely photometric and group parallaxes. But there they were required only for luminosity evaluation to an accuracy of a few tenths of a magnitude. In determining the mass of a visual binary, its parallax must be known to the same precision as $K_{1}+K_{2}$ for an eclipsing binary, i.e. to something like 5 per cent $( \pm 0.02$ in $\log \pi)$ for critical problems, as mentioned above. It is true that in relatively few cases are trigonometric parallaxes of visual binaries known to this precision, so the use of alternative methods should be encouraged. Eggen has chosen to employ these alternative methods in preference to trigonometric parallaxes smaller than 0.2 . With respect to photometric parallaxes, accuracy of 5 per cent in the orbital scale requires that the absolute magnitude obtained from the color be known to within o. I magnitude, an extremely stringent requirement. With respect to 'moving-group' parallaxes, difficulties of the method have been referred to in discussion of their application to the luminosities of eclipsing binaries, where the requirements are much less exacting. As examples of uncertainties in the moving-group parallaxes, one may refer to six cases discussed by Eggen (I), where two differing values of the published proper motions or small differences between two assumed velocity vectors give differences in $\log \pi$ of $0.04,0.04$, $0.06,0.08,0.09$, and 0.17 . These uncertainties are quite apart from the questions of membership in the groups and of ignoring considerable differences in the components of motion perpendicular to the galactic plane.

\section{EMPIRICAL DATA FROM BINARY SYSTEMS}

Data for visual binaries have been compiled in recent years by van de Kamp (rI), Harris, Strand, and Worley (12), and Eggen (I). The principal differences in outlook represented by these compilations are that Eggen includes double-star observations of only 'standard' observers, rejects trigonometric parallaxes smaller than $\mathrm{O}^{\prime \prime 2}$ (Harris, Strand, and Worley include parallaxes to $\mathrm{O}^{\prime \prime} \mathrm{O} 5$, van de Kamp to $\mathrm{O} . \mathrm{I}$ ), and adds stars with 'photometric' and 'moving group' parallaxes. I shall not comment further on the results from visual binaries.

Data for eclipsing binaries have been listed in recent years by Gaposchkin (r3), Wood (r4), and Harris, Strand and Worley (12). The last list referred to was intended to be restricted to the best determined properties. I have the following comments concerning their Table 3 :

I. The A star of $Z$ Vul fills its critical zero-velocity surface, and hence should not be included among 'normal' stars.

2. One or both components of $A R$ Lac and $Z$ Her are intrinsically variable by small amounts.

3. Improved values of the masses and radii of UV Leo are in press (6).

4. Colors of the components of TX Her and $\zeta$ Phe on a standard system are needed in order to obtain temperatures and $M_{\mathrm{bol}}$, or one of the alternative methods referred to should be employed. 
5. Conflicting results for the velocity amplitudes of $\mathrm{U} O \mathrm{ph}$ have been published by Plaskett (8) and by Abrami (9).

6. $M_{\mathrm{bol}}$ of all stars earlier than $\mathrm{B} 9$ are uncertain because of difficulties with the temperature scale.

7. See the list of stars given above, exclusive of types earlier than B8, which may eventually be added to the group of main-sequence systems with definitively determined properties.

Wood's and Gaposchkin's lists of absolute dimensions of eclipsing binaries are less restrictive than that of Harris, Strand, and Worley. Table 2 gives a brief comment on each system listed by Wood. For the $\mathrm{O}$ and $\mathrm{B}$ stars the comments are in addition to the problems mentioned for this class of binaries. The comment 'W UMa system' is intended to imply both that the stars are gravitationally bound in size and that the dimensions given are not trustworthy. A number of the additional systems of the much longer list by Gaposchkin may be expected to yield good results eventually, but none can at present be included in a critical list.

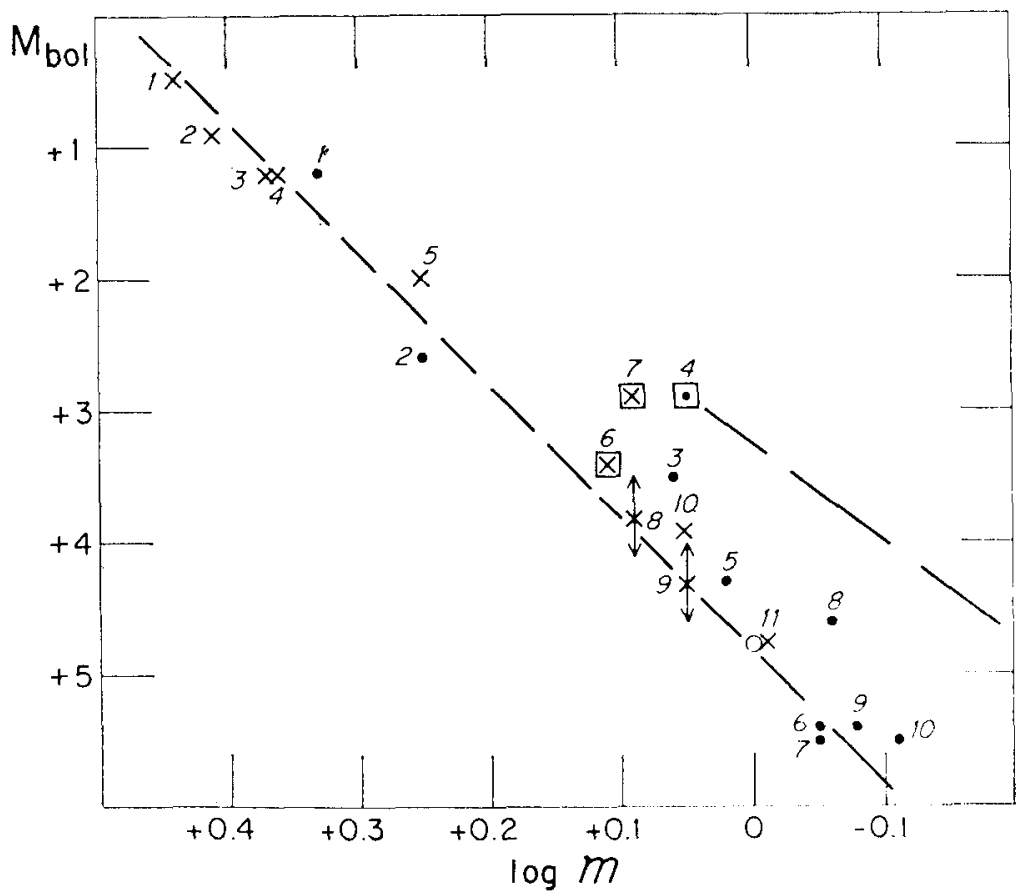

Fig. I. The mass-luminosity relation for well-determined systems of types $A$ to $G$. Dots: visual binaries; crosses: eclipsing binaries; squares: subgiants; circle: the Sun. The arrows show the range of luminosities permitted by the poorly observed light curve of $\mathrm{VZ}$ Hya. The lower line is the 'Sun-Sirius' relation and the upper the Hyades relation, both as given by Eggen.

A figure is shown (6) giving the mass-luminosity relation for eclipsing binaries with nearly equal main-sequence components later than B8 and for the best determined visual binaries. Added are the two best subgiants among the eclipsing systems. The lines shown are Eggen's 'Sun-Sirius' and Hyades mass-luminosity relations (r). To establish clearly whether a star 


\section{Table 2}

\section{Comments on the List of F. B. Wood}

\begin{tabular}{|c|c|c|c|}
\hline$\sigma$ & Aql & $\mathbf{B}_{3}$ & Eclipses too shallow for reliable inclination and radii. \\
\hline$\beta$ & Aur & A2 & Modern light curve needed. Eclipses shallow. \\
\hline$\zeta$ & Aur & $\mathbf{B}+\mathbf{K}$ & Complete revision in Astrophys. $\mathcal{F} .134,828,1961$. \\
\hline SX & Aur & $\mathrm{B}_{4}$ & Light curve unfavorable. \\
\hline $\mathrm{TT}$ & Aur & $\mathrm{B}_{3}$ & Photometry inadequate. \\
\hline WW & Aur & $\mathrm{A}_{7}$ & Good. \\
\hline AR & Aur & $\mathrm{Bg}$ & Good. \\
\hline $44 \mathrm{i}$ & Boo & G & W UMa system. \\
\hline UW & $\mathrm{CMa}$ & $\mathrm{O}_{7}$ & Light curve unfavorable. Velocities of fainter star uncertain. \\
\hline $\mathrm{AO}$ & Cas & $\mathrm{O}_{9}$ & Light curve very unfavorable. Difficulty with velocities of fainter. \\
\hline $\mathrm{U}$ & Cep & $B+G$ & Mass ratio very poorly determined. \\
\hline VW & Cep & $\mathrm{G}+\mathrm{K}$ & W UMa system. \\
\hline $\mathrm{AH}$ & Cep & Bo & Fair case. \\
\hline $\mathbf{U}$ & $\mathrm{CrB}$ & $\mathrm{B}_{5}$ & Velocity variation of cooler star completely unknown. \\
\hline RS & CVn & $F+G$ & Improved analysis in Astrophys $\mathfrak{F} .133,148,1960$. Fair case. \\
\hline 32 & Cyg & $\mathrm{B}+\mathrm{K}$ & Velocity variation of hotter star unknown. \\
\hline $\mathrm{X}$ & Cyg & Bo & Good. \\
\hline$V_{444}$ & Cyg & $\mathrm{WN}+\mathrm{O}$ & Difficult system. \\
\hline$V_{477}$ & Cyg & $\mathbf{A}+\mathbf{F}$ & Spectroscopic orbits under revision with higher dispersion. \\
\hline $3^{I}$ & Cyg & $\mathrm{B}+\mathrm{K}$ & Velocity variation of hotter star not well known. \\
\hline WW & Dra & $G+K$ & $\begin{array}{l}\text { Masses too large. Under revision. See also Eggen, Publ. astr. Soc. } \\
\text { Pacif. } 67,315,1955 \text {. }\end{array}$ \\
\hline YY & Eri & G & W UMa system. \\
\hline YY & Gem & $\mathbf{M}$ & Good. \\
\hline $\mathrm{Z}$ & Her & $\mathbf{F}+\mathbf{K}$ & Good. \\
\hline $\mathrm{RX}$ & Her & Ao & Good. \\
\hline $\mathrm{TX}$ & Her & $A+F$ & $\begin{array}{l}\text { Probably good. Verification of colors and velocity amplitudes } \\
\text { desired. }\end{array}$ \\
\hline $\mathrm{VZ}$ & Hya & $\mathbf{F}_{5}$ & Modern light curve needed. Masses good. \\
\hline SW & Lac & G & W UMa system. \\
\hline AR & Lac & $F+K$ & Good. \\
\hline CM & Lac & $\mathbf{A}+\mathbf{F}$ & Spectrum of secondary difficult. Under revision. \\
\hline UV & Leo & $\mathbf{G}_{2}$ & Improved analysis in press. Good. \\
\hline UX & Mon & $A+G$ & Difficult system. Results fair. \\
\hline $\mathrm{U}$ & Oph & $\mathrm{B}_{5}$ & Favorable system. Conflicting velocity amplitudes. \\
\hline ER & Ori & G & W UMa system. \\
\hline$A G$ & $\begin{array}{l}\text { Per } \\
\text { Phe** }\end{array}$ & $\begin{array}{l}\mathrm{B} 4 \\
\mathrm{~B} 6\end{array}$ & $\begin{array}{l}\text { Fair results. } \\
\text { Good system, although velocities of secondary difficult. }\end{array}$ \\
\hline$\mu_{1}$ & Sco & $\mathrm{B}_{3}$ & Light curve unfavorable. \\
\hline$V_{35} 6$ & Sgr & $\mathbf{B}+\mathbf{A}$ & Fair case. A star gravitationally trapped. \\
\hline W & UMa & G & W UMa system. \\
\hline $\mathrm{TX}$ & UMa & $B+G$ & Velocity variation of cooler star unknown. \\
\hline $\mathrm{AH}$ & Vir & $\mathbf{K}$ & W UMa system. \\
\hline$Z$ & Vul & $\mathbf{B}+\mathbf{A}$ & Fair case. A star gravitationally trapped. \\
\hline
\end{tabular}

* Note added in press: examination of the excellent series of Mount Stromlo spectrograms of $\zeta$ Phe shows that, as Hagemann has implied (Mon. Not. R. astr. Soc. 119, 143, 1959), the lines of the fainter component are so difficult to measure that the system should probably not be included among those with definitively determined properties. 
falls closer to one or the other of these relations requires the linear orbital scale to be known to about 5 per cent accuracy, as mentioned earlier. It is clear that for main-sequence stars later than B8, at any rate, significant information for the mass-luminosity relation will now be provided only by results of accuracy of the order referred to in the linear orbital dimensions. To emphasize this point, a figure is shown (6) illustrating the effects on the mass-luminosity relation

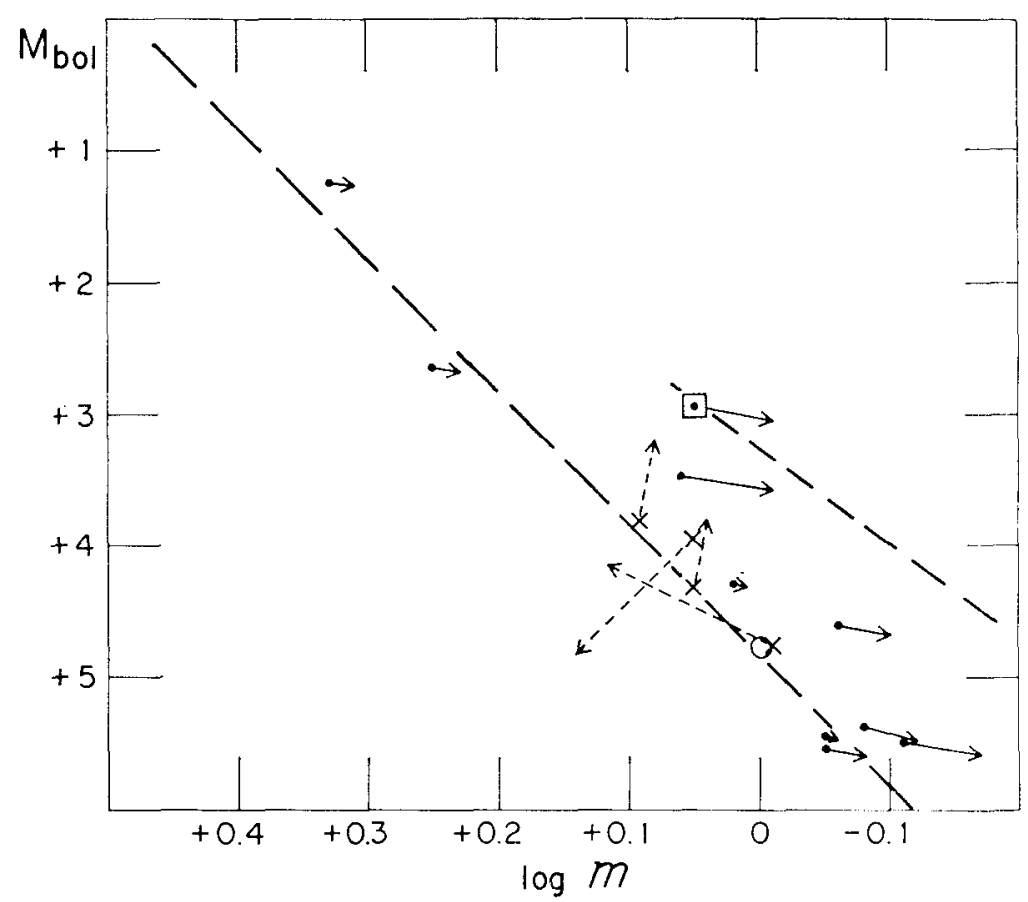

Fig. 2. Some of the stars of Fig. I. Solid arrows: visual binaries with parallaxes arbitrarily decreased by 0 "005; dashed arrows: older determinations of masses and luminosities of eclipsing binaries.

for the visual binaries of increasing each parallax arbitrarily by only o"005 (of the order of the systematic differences between long series of parallaxes at different observatories). Also shown are the 'old' and 'new' positions of three solar-type eclipsing binaries; refer to Table $\mathbf{I}$.

I turn briefly to the masses of cooler stars of luminosity class III or brighter. They present exceptionally difficult problems. Table 3 shows the present accumulation of data for the best cases. The ranges of masses given for the giant components of $\alpha$ Aur and TW Cnc indicate the uncertainties of the values.

\section{Table 3}

$\begin{array}{cccc}\text { Star } & \text { Type } & \text { Mass } & \text { Radius } \\ \alpha \quad \text { Aur } & \text { G8:III } & \mathbf{2} \cdot \mathbf{3}-\mathbf{3} \cdot \mathbf{I} & \text { 14: } \\ \text { TW Cnc } & \text { G8-Ko III } & \mathbf{2} \cdot \mathbf{3}-\mathbf{3} \cdot \mathbf{7} & \text { I0 } \\ \text { RZ Cnc } & \text { KI III } & 3 \cdot 2 & \text { II } \\ \phi \quad \text { Cyg } & \text { G8 III-IV } & \geqslant 2 \cdot 4 & - \\ \zeta \quad \text { Aur } & \text { K4 II } & 8 \cdot 3 \pm 1 \cdot 5 & \geqslant \text { I60 }\end{array}$


I commenced this talk by referring briefly to the impact Prof. Struve's ideas have had on our interpretation of the spectra of close binaries. I should like to conclude on a personal note. I had the great good fortune to have been associated with Prof. Struve, both at the University of Chicago and at the University of California, throughout my professional career until his death. I deem it a high privilege to be able to participate in this tribute to his memory.

\section{REFERENCES}

r. Eggen, O. J. Astrophys. F. Suppl., 8, 125, 1963.

2. Popper, D. M. Astrophys. F., 129, 647, 1959.

3. Popper, D. M. Astrophys. F., 129, 659, 1959.

4. Popper, D. M. Astrophys. F., 124, 196, 1956.

5. Popper, D. M. Astrophys. F., 133, 148, 1961.

6. Popper, D. M. Astrophys. F., r41, I965 (in press).

7. Popper, D. M. Astrophys. F., 134, 828, 196r.

8. Paskett, J. S. Publ. Dom. astrophys. Obs. Victoria, r, ז38, 1919.

9. Abrami, A. Mem. Soc. astr. Italiana, 29, 381, 1958.

I0. Ovenden, M. W. Mon. Not. R. astr. Soc. $126,77,1963$.

II. van de Kamp, P. Encyclopedia of Physics, Springer Verlag, Berlin, 50, 1958.

12. Harris, D. L., Strand, K. A., Worley, C. E. Basic Astronomical Data, University of Chicago Press, Chicago, 1963.

13. Gaposchkin. S. Encyclopedia of Physics, Springer Verlag, Berlin, 50, r958.

14. Wood, F. B. Basic Astronomical Data, University of Chicago Press, Chicago, r963.

\section{DISCUSSION}

Schwarzschild: I would like to emphasize the great value to the theory of stellar structure which Dr Popper's critical mass determinations represent. Particularly, the accurate masses for a few red giants, like Capella-first improved by Struve-and $\zeta$ Aurigae have played an enormous role for the theory of stellar evolution.

May I add a nasty question? Have you done absolutely everything possible to find one population II binary for a good mass determination? One such mass would give us a decisive clue to the initial He content of the Galaxy.

Popper: The burden of determining masses for population II stars falls actually on observers of visual binaries. Has not an eclipsing binary been discovered some years ago in $\omega$ Centauri? Unfortunately only a small percentage of eclipsing binaries yields masses of any degree of reliability.

Greenstein: I have attempted to find spectroscopic binaries among the halo population II $\mathrm{F}$ and $G$ subdwarfs: I have observed approximately 140 stars. From the radial velocities, I have only one definite spectroscopic binary, single-lined, with small amplitude and long period. There are three more suspected velocity variables, also small amplitude. In no cases are light variations yet known to exist.

Kraft: The reliable masses for giants contained in Popper's last slide seem all to have masses $>2 \odot$, whereas evolution would suggest that most giants come to the giant region along M 67-like tracks, and with masses $\sim \mathrm{I} \odot$.

Is this a result of a selection effect, a failure of the theory of stellar evolution, or what? 
Popper: The sample is very small to have conclusions of a statistical nature. I know of no selection effect that would work against finding giants of 1 or $1 \cdot 5$ solar masses.

Popper (additional comment): I neglected to point out that my results on solar type eclipsing binaries do not contradict Eggen's conclusion that the Sun-Sirius mass-luminosity relation is populated only by stars with appreciable ultraviolet excess.

\section{ON THE SYSTEM OF $\beta$ LYRAE}

\section{Sahade*}

During the war years, Prof. Struve engaged in an intensive programme of spectrographic observations of spectroscopic binaries, principally eclipsing variables. The choice of the subject was partly due to his inclination towards the study of double systems, evident from the very beginning of his work at Yerkes, but it was also due to the fact that, because of the war effort, the Yerkes Observatory was short of astronomers and Struve thought that he could help maintaining the output of Yerkes and McDonald by engaging in a subject which was certain to yield publishable results-at least orbital elements. We all know what the outcome was and where it led to.

Prof. Struve had a strong preference for the interpretation of systems that posed a challenge and as a consequence, peculiar close binaries were always in his observing programmes. Genesis and evolution of double stars and of planetary systems were the problems permanently present in the back of his mind.

As we know, we can distinguish several groups of systems that are peculiar in some ways and, in most cases, pose a problem of interpretation from the evolutionary point of view : the systems where one of the components is a Wolf-Rayet or an Of object, the Algol systems, the W Ursae Majoris systems, the cataclysmic variables, perhaps the metallic-line stars, perhaps also the $V \mid R$ variables, and the group with underluminous, massive components, to the existence of which $I$ have called attention recently ( $\mathbf{I})$.

In this paper I shall refer to $\beta$ Lyrae which belongs to the latter group. $\beta$ Lyr is an object, if not the object which merited more thought and effort from Prof. Struve than any other of the several hundred stars he investigated during his lifetime. As it is well known, $\beta$ Lyr has a period of about I 3 days, with no constant light at any phase of the orbital cycle. Its spectrum displays several sets of absorption as well as of emission features that arise from different sources; they have been described elsewhere (2). One of the sets of absorption lines arises from the primary component of the system, a B8 object of luminosity class II or III. Another set of absorption lines shows the effect of diluted radiation and originates in a gaseous envelope that surrounds the whole system, while broad absorption lines that are observed immediately before and immediately after mid-eclipse had been interpreted by Struve (3) as being the result of absorption effects of gaseous streams going from the secondary star towards the primary and from the latter component towards the former, respectively, seen projected upon the disk of the

* Member of the Carrera del Investigador Científico, Consejo Nacional de Investigaciones Científicas y Técnicas, Argentina. 